\title{
Summary: The Nucleus-A Close-Knit Community of Dynamic Structures
}

\author{
S. HENIKOFF \\ Howard Hughes Medical Institute, Fred Hutchinson Cancer Research Center, Seattle, Washington 98109 \\ Correspondence: steveh@fhcrc.org
}

\begin{abstract}
The 75th Cold Spring Harbor Symposium on Nuclear Organization and Function explored topics ranging from nucleosomes to nuclear pores. Exciting new genomic and imaging technologies have been used to characterize the nuclear interior, which consists of stable chromatin territories, dynamic domains, and self-organizing nuclear bodies. Histone variants and chaperones, posttranslational modifications, and ATP-dependent remodelers mediate nucleosome dynamics, regulated by Polycomb and other chromatin-associated proteins. Epigenetic memory is an emergent property of chromatin dynamics that is key to understanding how differentiated cells can become reprogrammed. Nuclear body composition and structure are becoming increasingly well understood, although their functions, if any, remain speculative. The nuclear envelope is strengthened by a fibrous lamin network that anchors chromosomes and represses gene expression, and disruption can lead to disease. Nuclear pores regulate the flow of substrates and products, using unstructured polypeptides to filter small molecules and flexible walls that allow large macromolecular assemblages to pass through. At mitosis, nucleosomes collapse into tightly packed nonfibrous cylinders that are then pulled to opposite poles at their kinetochores, where novel centromeric nucleosomes, mitotic motors, and spindle microtubules come together. By considering these complex processes in the context of the nucleus, the Symposium provided a coherent view of the genome in its native habitat.
\end{abstract}

It is fitting that this landmark Symposium, the 75th in the series, should be centered on the nucleus, where the genome meets cell biology. A day-long celebration, that took place just before the Symposium began, highlighted genomics and its impact on society, and the nucleus is a tangible embodiment of genomic processes. Attendees of this celebration were also treated to a publication by Jan Witkowski that recounted the history of this series, including personal recollections that reminded us of the impact that the Symposia have had on the history of our field and on the education of generations of scientists. The Cold Spring Harbor stamp was evident in the organization of the 75th Symposium by Bruce Stillman, Terri Grodzicker, David Spector, and David Stewart, whose insightful choices of topics and speakers and crafting of each session to tell a story resulted in an impressive program that was as much a course on the nucleus as it was a cross section of the most exciting research.

This was the first meeting on the nucleus per se, but numerous previous symposia have focused on processes that take place in the nucleus. In particular, the 1973, 1977, 1993, and 2004 meetings on chromosomes have established a tradition, and the reader is referred to Daniel Gottschling's Summary of the 2004 Symposium for a historical account of these previous conferences. The 38th Symposium on Chromosome Structure and Function provides a midway point between the first Cold Spring Harbor Symposium in 1933 and the current one. That 8-day event in 1973 was especially memorable for me, as a starting graduate student attending my first scientific meeting ever. It took place shortly before nucleosomes were shown to be the basic units of packaging of the eukaryotic genome. Harold (Hal) Weintraub, a speaker in 1973, summarized the 1993 Sym- posium, in which he recounted the enormous progress made during the intervening 20 years in working out the biochemistry of chromatin. However, our understanding of the relationship between chromatin and development remained fragmentary, and Hal closed his Summary by noting that there seemed to be only "local problems and local solutions," cautioning readers not to expect any emerging principles. Since then, enormous progress in genomics and technology in general has resulted in a global perspective of nuclear organization and function. Below, I summarize several themes that emerged during the 5 days of talks, posters, and informal discussions that made the 75th Symposium an especially satisfying experience.

\section{EPIGENETICS FROM NUCLEOSOMES TO REPROGRAMMING}

The reprogramming of differentiated adult cells into induced pluripotent stem cells by expression of transgenes has captured intense public attention (Hockemeyer and Jaenisch, this volume). However, transgene induction of developmental reprogramming is not a new approach. Twenty years earlier, Hal Weintraub and colleagues had demonstrated that nonmuscle cells could be reprogrammed to make muscle by expression of the master regulator of muscle development, MyoD (Davis et al. 1987). Long before that, John Gurdon et al. (1971) had described reprogramming by nuclear transfer, and the Xenopus system that he used in his classic work continues to yield new insights. In the opening talk of the meeting, Gurdon described how a change in the developmental fate of transplanted Xenopus nuclei can be inherited during many rounds of division without ongoing transcription and how 
this system was used to identify the histone replacement variant H3.3 as mediating epigenetic memory. Histone replacement with H3.3 during germ-cell development occurs in organisms as diverse as mammals, flies, worms, and plants (Ingouff et al. 2007; Ooi and Henikoff 2007), which implies that to reprogram a nucleus, the genome must renew itself by replacing existing chromatin proteins with new ones, often in rapid fashion.

There are only four amino acid differences between H3.3 and its replication-coupled H3 counterpart, and antibodies have not been useful for separating H3.3 and H3 nucleosomes. Transformation with epitope-tagged transgenes has been the norm, but David Allis described the use of zinc-finger targeting to replace an endogenous H3.3 gene with tagged derivatives in mammalian cells (Elsaesser and Allis, this volume). H3.3 profiles track with transcriptionally active genes and regulatory elements, but surprisingly, the loading machinery differs among classes of sites (Elsaesser and Allis, this volume). HirA is the H3.3 chaperone for gene body incorporation, but the DAXX protein, together with the ATP-dependent nucleosome remodeler ATRX, replaces H3 with H3.3 at regulatory elements and telomeres. This division of labor among histone chaperones reveals a new paradigm for achieving specific localization of a particular variety of chromatin. An intriguing finding was that H3.3 is unevenly distributed in brain, which makes one wonder about the extent to which neural memory and epigenetic memory use the same underlying chromatin-based mechanisms.

Polycomb-group protein complexes perpetuate the epigenetically silent state in animals and plants. The PRC2 complex maintains H3K27 methylation, and Danny Reinberg provided evidence for a model in which PRC2 recognizes H3K27me3 on one nucleosome while methylating an adjacent nucleosome (Margueron et al. 2009). In both flies and mammals, binding by the PRC1 complex mediates silencing (Ringrose and Paro 2007). It has generally been assumed that silencing itself is mediated by histone modification, either $\mathrm{H} 3 \mathrm{~K} 27 \mathrm{me} 3$ or ubiquitylation of $\mathrm{H} 2 \mathrm{~A}$ or H2A.Z (Kallin et al. 2009). However, recent studies reported at the Symposium indicated that whereas H3K27 methylation facilitates targeting of PRC1, compaction of the chromatin, not histone modification, mediates silencing (Eskeland et al., this volume; Grau et al., this volume). Nucleosomes at sites of Polycomb protein binding turn over more slowly than at sites of corresponding loci that are not bound by PRC1 (Deal et al. 2010), as if silencing is a direct result of the loss of mobility caused by increased chromatin compaction. It appears that a positively charged disordered domain of a PRC1 complex component is responsible for compaction, and this domain has shifted from one to another PRC1 subunit during Drosophila evolution (Grau et al., this volume).

\section{HETEROCHROMATIN: NOT JUST A BAD NEIGHBORHOOD}

The 1973 Symposium took place just before recombinant DNA cloning became the dominant molecular biology tool, and thus the most practical means of studying chro- mosomal DNA was to isolate tandem repeats, because these were the only sequences that could be obtained in pure form. Gel-based DNA sequencing was still years off; nevertheless, at that meeting, Joseph Gall reported the 7bp sequences of all three Drosophila virilis satellite DNAs, which account for $\sim 40 \%$ of the D. virilis genome (Gall et al. 1974). Furthermore, Gall sequenced both strands, which in retrospect may have been the first direct proof of the self-complementarity of DNA.

The dominant methodology for gene analysis in 1973 was renaturation kinetics, which led to the identification of what were later referred to as long interspersed elements (LINEs) and short interspersed elements (SINEs) (Singer 1982). At first, these elements were misunderstood, and only later was it revealed that interspersed repetitive elements correspond to transposable elements, leading to a much fuller appreciation of Barbara McClintock's pioneering studies of jumping genes in maize (McClintock 1951). Although she thought of transposons as controlling elements, they are unquestionably dangerous genomic parasites that host systems have evolved to silence. Despite the best damage-control efforts of the host genome, an occasional transposon becomes domesticated, sometimes as a controlling element. For example, the regulatory circuitry of early mammalian development is conserved, but repeat-associated binding sites shift among species, some $25 \%$ of which result from transposition (Heng et al., this volume).

The best defense against transposons is to silence them so that they cannot transpose to new sites, most critically in germ cells, where insertions will be transmitted to future generations. Robert Martienssen paid homage to McClintock in describing the elaborate process whereby siRNAs defend against such genomic parasites in germ cells (Martienssen et al., this volume). In Arabidopsis thaliana, 21-nucleotide dsRNAs are made in the vegetative cell and move into the sperm cells within the pollen grain, a paradigm for siRNA-mediated cell-to-cell communication. It seems likely that a similar process is occurring on the female side to silence transposons in the egg cell genome, and it is intriguing to think that this process, elaborated first in plants, as in so many other cases, will also be found to occur in mammalian primordial germ cells (PGCs). Although siRNA-mediated targeting of heterochromatin formation has thus far been limited to plants and fungi, Gary Felsenfeld presented evidence that a 16$\mathrm{kb}$ block of heterochromatin, which borders the chicken $\beta$-globin gene and helps to insulate it from a neighboring locus, requires the Dicer-Ago2 siRNA system (Giles et al. 2010). This finding suggests an in vivo function for RNAi, which has been one of the most important reverse genetic tools in biology, in vertebrates.

RNAi-mediated heterochromatin formation has been elucidated in detail in Schizosaccharomyces pombe, where it functions in part to create a hypoacetylated environment in the pericentromeric repeats conducive to deposition of CenH3 (CENP-A) nucleosomes in the central core, where the kinetochore assembles (Lejeune et al., this volume). There also seems to be another pathway for heterochromatin formation in S. pombe that does not depend on dou- 
ble-strand RNA (Aygün and Grewal, this volume). Nuclear retention of RNA transcribed from repeats in the nucleus would impede RNA polymerase II (Pol II) at heterochromatic sites, and this attenuation process would facilitate silencing. How excess RNA would interact with repeats to impede Pol II is not clear, but an attractive possibility is that it forms triplexes with duplex DNA similar to the triplexes that are responsible for targeting DNA methylation at ribosomal DNA cistrons (Bierhoff et al., this volume). Could this be a general mechanism for creating heterochromatic patches within genes? It is interesting that local HP1 binding within genes had been seen previously in mammals (Vakoc et al. 2006) and flies (de Wit et al. 2007). The slowing down of Pol II by less dynamic chromatin that results from HP1 binding might allow for one or another exon junction to be chosen.

Slowing down of Pol II is also associated with alternative splicing in mammals and fission yeast (Allo et al., this volume; Waks and Silver, this volume). An S. pombe mutant Pol II was introduced to slow down transcription, and slower elongation through an intron resulted in a switch in splicing preference. The mapping of Agol genome wide provided correlative evidence that it targets histone methylation and suggested transient formation of a heterochromatic patch (Allo et al., this volume). Jim Haber summarized the discussion:

\section{Methylation of histone tail's K \\ Stops splicing of some RNA. \\ But it's not copasetic \\ To claim epigenetic \\ If it fluctuates during the day.}

Rather than transient epigenetic silencing, it is possible that increases in histone methylation are direct consequences of slowing down Pol II-mediated chromatin disruption. Consistent with this possibility, there are associations among Pol II transcriptional elongation, histone modifications, and splicing efficiency in budding yeast, which lacks "repressive" histone methylation (Buratowski, this volume). Perhaps tethering of histone methyltransferase complexes to the Pol II carboxy-terminal domain in $S$. pombe leads to an increased window of exposure to nucleosomes during disruption by transcription, and this would inevitably result in increased histone methylations of various types at steady state. This scenario could explain why the correlations between the Ago1 RNAi-associated Argonaute protein and methylations associated with heterochromatin were no better than those for H3K36 methylation, which is associated with transcriptional elongation, not with silencing.

Another unexpected mode of heterochromatin formation in S. pombe was described by Nicholas Proudfoot. Convergent transcription is rare, yet all three genes encoding the RNAi machinery reside in convergent pairs, and these are down-regulated during S phase (Gullerova and Proudfoot, this volume). It appears that read through occurs only when genes are convergent, and heterochromatin results. How does this happen? One possibility is that the positive supercoiling stress that builds up as RNA polymerases approach one another slows them down, perhaps involving an RNA retention/triplex formation process. Tandem genes do not show this mode of heterochromatin formation, which implies an epigenetic transcriptional terminator.

Renaturation kinetics, or $\mathrm{C}_{0} \mathrm{t}$ curve analysis, made a comeback at the 75th Symposium. Cot1 DNA is the highly repetitive fraction that has been used to suppress cross-hybridization of repeats for in situ hybridization. Jeanne Lawrence described how Cot1 transcripts are present over condensed euchromatic blocks but absent over the Barr body, which is the mammalian female inactive $\mathrm{X}$ chromosome that is maintained in a condensed state by the Xist long noncoding RNA (Lawrence et al., this volume). Perhaps Xist mediates coalescence of the Cot1 DNA repeats, and the compaction itself would inhibit transcription of Cot1 DNA sequences.

Lawrence also showed that, in contrast to expectation, genes are absent from the Barr body whether or not they are silenced or escape inactivation but, rather, neatly decorate the periphery (Lawrence et al., this volume). This configuration is reminiscent of amphibian lampbrush chromosomes, which were still a favorite model system in 1973 (Pardue 1974). Remarkably, this configuration seems to be general, because both David Spector and Andrew Belmont described peripheral transcription around the core of a loop for repeated gene arrays (Belmont et al., this volume; Hübner and Spector, this volume), and Thomas Cremer reported a similar situation for ordinary single-copy genes, where the transcribed loops reside in the perinuclear space (Markaki et al., this volume).

\section{THE DYNAMIC NUCLEUS}

The nuclear pore complex (NPC) would seem to be the least dynamic of all nuclear structures, but as Günter Blobel described it, the NPC is a highly flexible construction, with walls that can distort as much as $10 \mathrm{~nm}$ to allow large macromolecular complexes to pass through (Blobel, this volume). On the outside, unstructured protein repeats resemble tentacles that wave in cytoplasmic currents, filtering out proteins that lack nuclear localization signal sequences. The computed map of this beautiful eightfold symmetric 70-Mda structure reveals the locations of the many scaffold proteins as well as the peripheral nucleoporins (NUPs) (Tetenbaum-Novatt and Rout, this volume). Whereas the scaffold proteins are as stable as the nuclei of which they are part, the peripheral NUPs are highly dynamic and move between the pore and chromatin, where they have roles in gene regulation (Capelson et al., this volume). Transport is dynamic going the other way as well. Robert Singer described how mRNAs encounter a traffic jam going into the pore and zip quickly through the pore on their way out, where a ribosome is waiting (Lionnet et al., this volume).

The lamin filaments must be stable enough structures to give the nucleus its spherical shape. A-type lamins are expressed during later stages of development and are responsible for an astonishing variety of phenotypes, including loss-of-function mutations that interfere with cell cycle regulation (Dechat et al., this volume). Gain-of- 
function mutations in A-type lamins cause accelerated aging, failure to maintain stem cell populations, resistance to cancer (Scaffidi and Misteli, this volume), and elaborate distortions of the nuclear envelope (Shimi et al., this volume), as succinctly described by Jim Haber:

The functions of lamins are curious.

Even simple mutations are serious.

Cells quickly die

With lobed nuclei

Not to mention infarcts and progerias.

Whereas lamins are stable during interphase, lamin-associated chromatin is dynamic. Bas van Steensel described a novel technique for imaging this process in vivo by tethering Dam methylase to a lamin to mark lamin-associated sites, followed by detection using a methyladenine-binding domain. Remarkably, lamin-associated chromatin underwent limited short excursions into the interior, demonstrating that the associations of chromatin with lamin are dynamic (Peric-Hupkes and van Steensel, this volume). This might help to account for how as much as half of the chromatin can be associated with lamins, just not all at the same time. No doubt, this in vivo chromatin marker will be widely used to characterize other dynamic nuclear structures. Genome-wide mapping of lamin associations during mammalian development (Peric-Hupkes and van Steensel, this volume) and imaging of a large lamin-associated repeat during worm development (Towbin et al., this volume) left little doubt that release of associations from the nuclear envelope into the interior is a mechanism for gene activation. The engineering of a lamin muscular dystrophy in worms was a persuasive demonstration of the value of a simple model organism to gain a molecular understanding of human disease (Towbin et al., this volume).

There is a growing appreciation of the importance of nucleosome dynamics in maintaining totipotency and pleuripotency, and primordial germ cells are natural examples in which reprogramming requires global chromatin changes. Azim Surani described studies that narrowed down the global process of demethylation followed by large-scale remodeling to a 4-h period in PGCs (Surani and Hajkova, this volume). Evidence was presented that the base-excision repair (BER) protein $\mathrm{XRCC} 1$, that binds to single-strand breaks, is present during the short period in which demethylation appears. Cleavage and base removal during BER would seem to be a dangerous process, considering that there are 10-20 million methylated base pairs in a mammalian genome. Perhaps the large majority of 5-methylcytosines are oxidized to 5-OH methylcytosine, and BER occurs only at a handful of sites that are missed. Consistent with this possibility, the Tet1 5-methylcytosine oxidase is up-regulated at this critical time (Surani and Hajkova, this volume). The removal or oxidation of 5-methylcytosine is followed by massive chromatin remodeling, including loss of $\mathrm{H} 1$ and H2A.Z. PolyADP ribose polymerase (PARP) evidently replaces $\mathrm{H} 1$ to help to rapidly turn over nucleosome cores to achieve totipotency.

PARP has also been implicated in transcriptional elongation. John Lis described a live imaging system for de- tecting changes on a timescale of seconds, showing chromatin being disrupted even before Pol II passes through (Guertin et al., this volume). PARP catalyzes the growth of polyADP ribose chains, and Lis proposed a model in which PAR polymers have a direct role in compartmentalization of transcription, perhaps by forming a transient cage that defines the transcriptional compartment (Zobeck et al. 2010).

More common mechanisms for regulating transcriptional elongation include replacement of $\mathrm{H} 2 \mathrm{~A}$ with $\mathrm{H} 2 \mathrm{~A} . \mathrm{Z}$ (Watanabe and Peterson, this volume) and pausing of Pol II (Newman and Young, this volume). Release of paused Pol II by the Myc oncogene may be a general mechanism for regulation of transcriptional elongation in mammals and might help to explain why Myc overexpression leads to cancer. Whether what is measured as pausing is actually premature termination (Buratowski, this volume) remains unclear, maybe even to Pol II itself, as Jim Haber pointed out:

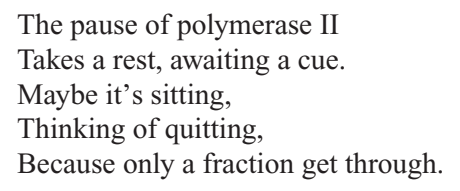

Nucleosome turnover is also a key process at active genes and regulatory elements, which are far more dynamic than had been appreciated in the past. ATP-dependent remodelers have been implicated in driving turnover, but how do they work? Craig Peterson described a system involving two ATP-dependent nucleosome remodelers, which work in opposite directions on histone dimers (Watanabe and Peterson, this voulme). The Swr1 complex replaces $\mathrm{H} 2 \mathrm{~A} / \mathrm{H} 2 \mathrm{~B}$ with $\mathrm{H} 2 \mathrm{~A} . \mathrm{Z} / \mathrm{H} 2 \mathrm{~B}$, and the INO80 complex performs the reverse reaction. This would seem to be a futile cycle, except that continuous replacement of nucleosomal heterodimers will transiently expose DNA to make it accessible. Another potential mechanism for making regulatory DNA more accessible for activation was described by Shelley Berger: In response to stress, AMP kinase moves into the nucleus, where it phosphorylates histone $\mathrm{H} 2 \mathrm{~B}$ on serine 36 , likely initiating gene activation (Bungard et al. 2010).

\section{REPEATS AND REDUNDANCY}

A major quest in 1973 was to resolve the C-value paradox, which refers to the fact that genome size often does not correlate with organismal complexity. Some salamanders have one order of magnitude more DNA than some frogs, and some amoeba have orders of magnitude more DNA than do we. Numerous speculations were put forward, redundancy being the favorite. For example, it was known that most tandemly repetitive satellite DNA can be deleted, and the $40 \%$ of the $D$. virilis genome that Gall first sequenced is an example of what was considered to be “junk" DNA (Gall et al. 1974). Other long tandem repeats express ribosomal RNA, and in 1973, these rDNA arrays were among the few sources of transcriptionally active genes that could be isolated for molecular study (Brown and Sugimoto 1974). Expression of even a single rDNA gene leads to the self-organization of a recognizable nu- 
cleolus (Karpen et al. 1988), the first example of a nuclear body. Other such nuclear bodies were described by Santiago Ramón y Cajal more than a century ago. Understanding the molecular basis for nuclear body self-organization has long been a goal for cell biologists (Misteli 2001).

At the 75th Symposium, Gall delivered the Reginald Harris lecture, describing his latest studies of Cajal bodies, which, unlike nucleoli, are not directly associated with chromatin (Nizami et al., this volume). Using a mutation in coilin, the major protein constituent of Cajal bodies, Gall showed that complete loss of Cajal bodies has no detectable phenotypic effect. Nonessentiality is not a case of redundancy, because the small nuclear ribonucleoproteins (SNURPs) and other components that are packed into Cajal bodies seem to behave normally even if they are not concentrated in a sphere. There is a tendency for graduate students to gravitate toward problems that they deem to be important, but Gall has had enormous success throughout his career by studying nuclear constituents that apparently have no function at all.

Unlike Cajal bodies, coilin-containing histone locus bodies (HLBs) are tethered to the tandem histone repeats and require the NPAT histone gene transcription factor to form (Rajendra et al., this volume). NPAT mutations survive through most of Drosophila development, and there are no detectable effects of HLB absence on histone mRNA maturation. As Jim Haber concluded, the function of coilin-containing nuclear bodies, if any, remains unresolved:

Histone bodies and those of Cajal

Wrap up mRNAs into a ball.

If N-PAT is gone

The bodies have flown

But trimming's not altered at all.

During the symposium, David Spector proposed that the high concentration of components associated with abundant transcription of closely spaced tandem repeats might result in a dynamic structure surrounding a point source. In this way, HLBs and other spherical nuclear bodies, such as the perinucleolar compartment that forms in cancer cells (Slusarczyk et al., this volume) and induced loci that recruit high levels of the JMJD3 histone demethylase (Hübner and Spector, this volume), would cytologically resemble transcription of artificial arrays that have been widely used in live cell studies of chromatin dynamics (Belmont et al., this volume).

Whereas cells can thrive without these nuclear bodies, origins of replication occupy the other extreme on the essentiality scale. A sufficient number of dispersed replication origins are required in a cell so that the entire genome can be replicated before mitosis ensues. One idea regarding the evolution of eukaryotes is that they were able to tolerate an unlimited expansion of their genomes once they evolved a way of quickly replicating it all, and multiple origins of replication on a chromosome were the solution. Three redundant systems have evolved to prevent origins from refiring before completion of the cell cycle, a fail-safe strategy to avoid the disastrous situation that would occur if even one replication origin were to misfire (Diffley, this volume).
The C-value paradox also had an RNA aspect, in that far more of the genome is transcribed than ends up on polysomes and is translated in the cytoplasm. This socalled heterogeneous nuclear RNA (hnRNA) lent itself to many speculations, all wrong. The discovery that genes are organized into exons and introns was a major surprise when it was reported at the 1977 Cold Spring Harbor Symposium (Chambon 1978), and the roles of most classes of noncoding RNAs have since been elucidated. However, the small noncoding HSUR RNAs produced by herpesvirus are not involved in viral assembly, and their function has long been elusive. Remarkably, HSURs appear to neutralize host microRNA (miRNA) antiviral defenses, an example of genetic conflict between one small noncoding RNA and another (Cazalla and Steitz, this volume).

For decades, it was assumed that splicing explains hnRNAs and the C-value paradox. Nevertheless, careful annotation of the human genome has led to the detection of an estimated 10,000 long noncoding RNAs (lnRNAs), which continue to resist explanation (Ørom et al., this volume). InRNAs are mostly cell-type specific, expressed at very low levels, and have chromatin features that are characteristic of transcribed genes. A few lnRNAs have been tested for function and were found to enhance expression of genes that are nearby or not so nearby. The human genome was declared finished about a decade ago, but it seems that we are still a long way from deciphering its intricacies.

\section{IT'S FRACTAL!}

Imaging technology in 1973 was crude, as noted by Hewson Swift in his Summary of the 38th Symposium: "Spread whole chromosomes under the electron microscope look even at their best something like a bad day at a macaroni factory." But we now have genome-based methods that can potentially resolve chromatin folding within the nucleus: Coupling of the $3 \mathrm{C}$ (chromosome conformation capture) method (Dekker et al. 2002) to deep sequencing revealed that the human genome does not resemble Swift's bad day at all (Lieberman-Aiden et al. 2009). Unlike cooked pasta, which becomes tangled when cooked, the genome resembles ramen noodles, which are "dense, but totally unentangled, so you can pull out a noodle or a bunch of noodles without disrupting the rest." (Erez Lieberman-Aiden, quoted in Discover magazine, October 12, 2009). Technically, this topological arrangement is referred to as a fractal globule, which is consistent with electron microscope (EM) observations showing that mitotic chromosomes can also be modeled as fractal structures (Maeshima et al., this volume).

A lesson here is that genomic technologies have the potential to close the wide gap between DNA sequence-level resolution and imaging of tightly packed chromatin in the nucleus. Global interaction maps can also provide insights into other nuclear processes that have previously been studied only in one dimension. A striking example of this was presented by David Gilbert, whose early-versus-late replication map of the human genome nearly coincided with the genome interaction map (Gilbert et al., this vol- 
ume). The mechanistic basis for this correspondence of the genome in time and space is unknown; however, it seems reasonable to suppose that that the $\mathrm{G}_{1}$ timing decision point described by Gilbert, which is a measure of chromatin maturation, has a three-dimensional (3D) interpretation.

Regular fibers of 30-nm width are readily isolated from cells and can be produced from reconstituted chromatin in vitro, but there has been a long-running debate regarding their existence in vivo. For example, an estimation of the mean contour length of yeast chromatin from $3 \mathrm{C}$ data implies that chromatin fibers are too extended to be $30 \mathrm{~nm}$ in width (Dekker 2008). Better EM methods now provide direct tests of the 30-nm fiber model in whole cells. David Bazett-Jones described electron spectroscopic imaging of nuclei to image nitrogen and phosphorus as a means of visualizing DNA and protein separately (Fussner et al., this volume). Only $10-\mathrm{nm}$ fibers were seen in thin sections, with broad spaces in between, and no 30-nm fibers were detected. Traditionally, the 30 -nm fiber was invoked to explain heterochromatin, and the fact that $30-\mathrm{nm}$ fibers are present in avian erythrocytes and in sperm fits with that paradigm. It is only in active nuclei that 30 -nm fibers appear not to exist, and as noted by Jim Haber, we need to focus more on the how and the why:

How do fibers of chromatin fold?

"It's fractal"! "It's spongy"! we're told.

It's all very strange

That neighborhoods change

But how are these movements controlled?

What about mitotic chromosomes? Cryo-EM images failed to show the presence of any fibers at all but, rather, appear to be totally disordered (Eltsov et al. 2008). This conclusion was confirmed by X-ray scattering, which also suggested that previous detection of a 30 -nm feature in Xray scattering data could be accounted for by contamination with ribosomes (Maeshima et al., this volume). The resulting model of the metaphase chromosome is of an interdigitated polymer "melt," characterized by dynamic local movement of closely packed nucleosomes and mitotic proteins.

A fractal structure such as a mitotic chromosome should coalesce into a sphere as it collapses, and thus it is mysterious that unstructured chromatin condenses into cylindrical mitotic chromosomes. Andrew Belmont described progress with large repeated arrays that self-organize into subchromosomal structures and might be responsible for breaking the symmetry and yielding cylindrical chromosomes with relatively uniform diameters that are independent of length (Belmont et al., this volume). An alternative possibility is that the nucleosomes themselves are modified in such a way that cylindrical structures form, and Daniela Rhodes described how ubiquitylation of H2A yields highly elongated fibers. Another possibility was suggested by the findings of Kenneth Zaret, who described shifting of FoxA during chromosome condensation (Zaret et al., this volume) - this might result in a configuration that nucleates cylindrical packaging of mitotic chromatin. Other proteins that remain with chromosomes during mitotic condensation likely have similar roles, and their detection will be aided by quantitative mass spectrometry that William Earnshaw showed has identified essentially all of the proteins in chicken mitotic chromosomes (Ohta et al., this volume).

Until recently, EM was required to observe nucleosomes because the required level of resolution exceeds the Abbe diffraction limit for light. However, Thomas Cremer described the application of two new 3D fluorescence methods, structured illumination microscopy and spectrally assigned localization microscopy, that yield images with awesome clarity, in which individual nucleosomes appear as separated dots (Markaki et al., this volume). Cremer also described high-resolution live fluorescent imaging. Photoactivation of patterns drawn onto nuclei labeled with histone GFP, followed by live imaging, demonstrated that chromatin compartments are globally stable from $G_{1}$ through $S$ phase to $G_{2}$, are disrupted during mitosis, and reform in daughter nuclei.

\section{GENES KISS AND TELL}

Looping has emerged as the most likely mechanism for long-range interactions between enhancers and promoters, and the subdivision of chromosome territories into much smaller compartments increases the probability of a transcription factor making productive contact with its promoter. However, there have been many observations of interactions among chromosomes, and reports at the meeting described some of these.

The "transcription factory" concept is based on the notion that moving DNA through a fixed polymerase is a more efficient solution to the challenge posed by a double-strand DNA template that twists $360^{\circ}$ every 10 bases, the alternative being to spin RNA polymerase and its associated complexes (Kimura et al. 1999). Fixing polymerases also might permit their coalescence, where high concentrations of components result in more efficient RNA synthesis, and Peter Fraser described examples in which genes regulated by a common transcription factor closely approach within factories, even when located on different chromosomes (Eskiw et al., this volume). The proximity of sites in factories can help to explain the preferential location of breakpoint pairs found in cancer.

Direct contact among loci might also be involved in the coordination of multiple chromatin regulators by the mammalian $H 19$ locus, where application of $4 \mathrm{C}$, a genome-wide version of $3 \mathrm{C}$, detected up to seven sites simultaneously associated with $H 19$ (Göndör et al., this volume). Similarly, "kissing" of a long-range regulatory element within the Drosophila Bithorax complex with distant sites along the chromosome is thought to pull genes into compartments where they are maintained in the off state by Polycomb proteins (Cheutin et al., this volume). Cancer is associated with large-scale changes in nuclear positioning, although these changes do not appear to be responsible for gene silencing by aberrant DNA methylation (Easwaran and Baylin, this volume). It will be interesting to determine whether such long-range contacts are mediated by lnRNAs that have been described for some of these and other loci (Hung and Chang 2010). 
The best-studied lnRNA is Xist, which is produced at the $\mathrm{X}$-inactivation center $(\mathrm{Xic})$ and is responsible for $\mathrm{X}$ chromosome inactivation. Edith Heard described a live imaging analysis of cells acquiring asymmetry in the process of one of the $\mathrm{X}$ chromosomes becoming silenced by Xist and the other remaining active (Heard, this volume). This process involves a nearly hour-long period of pairing, in which the Xics are relatively close, with occasional closer encounters that would presumably correspond to kissing and an asymmetric transfer resulting in only one Xic becoming inactivated.

Despite considerable progress, the molecular mechanisms responsible for interactions among nuclear compartments remain mysterious. As Thomas Cremer quipped, "We and others have obtained compelling evidence that we do not understand this problem."

\section{MITOTIC MIFFS}

During the 42nd Symposium in 1977, Ulrich Laemmli described a condensed structure visible by EM that appeared to anchor DNA loops along the length of mitotic chromosomes. This discovery of a putative mitotic scaffold encouraged efforts to characterize an interphase counterpart, the nuclear matrix, that was hypothesized to provide structural support for the nucleus and serve as a guide for channeling substrates and products (Capco et al. 1982). However, interest in the controversial nuclear matrix has since waned, and it was rarely mentioned during the 2010 Symposium. Nevertheless, debates regarding the mitotic scaffold continue (Belmont et al., this volume), and the need to explain how a fractal globule without any detectable fibrous structure can collapse into a cylinder rather than a sphere (Eltsov et al. 2008) seems to support the existence of a mitotic scaffold of some kind, the composition of which remains unknown.

Meanwhile, new discoveries have fueled new controversies, some of which center around the functioning of structural maintenance of chromosome (SMC) proteins that are involved in a variety of processes, including twofold repression of hermaphrodite X chromosomes responsible for Caenorhabditis elegans dosage compensation (Meyer, this volume). Sister chromatids are held together by an SMC protein ring structure, cohesin, that is cleaved by the separase protease to release sister chromatids from all chromosomes simultaneously at the anaphases of mitosis, meiosis I, and meiosis II (Nasmyth and Oliveira, this volume). Remarkably, this same cohesin/separase system has evolved to release sister centrioles at the same time as sister centromeres, thus ensuring that there is one centriole at each pole to orient the mitotic spindle for every genome that will be pulled to it (Hatch and Stearns, this volume). The topology of the cohesin ring has been a matter of debate. Kim Nasmyth presented evidence in favor of his model that a single ring encircles both DNA gyres, as opposed to two fused rings, each surrounding a single gyre (Nasmyth and Oliveira, this volume).

A new debate was sparked by Luis Aragón's description of the mitosis-associated removal of residual sister-chromatid catenations that are not removed during replication
(Baxter and Aragón, this volume). Most newly replicated sister chromatids are decatenated behind the replication fork by the SMC5/6 complex that evidently senses DNA topology (Kegel and Sjögren, this volume). In the absence of the condensin SMC complex, removal of residual catenations fails (Baxter and Aragón, this volume). Bruce Stillman suggested that a similar failure might account for the pre-anaphase arrest that occurs in the absence of origin recognition complex (ORC) subunits ORC2/ORC3 that reside around centromeres during mitosis (Stillman et al., this volume). As described by Aragón, yeast circular minichromosomes remain concatenated in the absence of topoisomerase II when they are pulled to the poles and undergo a remarkable reversal of DNA supercoiling from minus to plus. A suggestion that the tension would be so extreme that the nucleosomes might be popping off seems implausible, because this would result in loss, not reversal, of supercoiling. A related possibility is that tension pops off just the $\mathrm{H} 2 \mathrm{~A} / \mathrm{H} 2 \mathrm{~B}$ dimers, transiently leaving behind a symmetrical $(\mathrm{H} 3 / \mathrm{H} 4)_{2}$ tetramer that is known to wrap DNA in either a right- or a left-hand orientation (Hamiche and Richard-Foy 1998).

The directionality of supercoiling of yeast minichromosomes was also a factor in a controversy concerning the composition and structure of the centromere-specific nucleosome that contains CenH3 in place of H3. We had reported that supercoiling reverses when $\mathrm{H} 3$ nucleosomes are replaced by $\mathrm{CenH} 3$, both for Drosophila CID in vitro and for yeast Cse4 in vivo (Henikoff and Furuyama, this volume). The right-hand wrap implied by this observation is consistent with either "hemisomes" of CenH3/H4/H2A/ $\mathrm{H} 2 \mathrm{~B}$ or "tetrasomes" of $(\mathrm{CenH} 3 / \mathrm{H} 4)_{2}$, but not with octamers. Don Cleveland described how right-hand wrapping is incompatible with evidence for unconventional human CENP-A octameric nucleosomes assembled in vitro, which are left handed (Conde e Silva et al. 2007; Sekulic et al. 2010). Also fueling this debate was evidence presented by Stephen Harrison, who described the 3D structure of the Ndc10 protein, which is essential for stabilizing the yeast $\mathrm{Cse} 4$ nucleosome in vivo (Cho et al., this volume). The parallel orientation of the two DNA-binding domains in the Ndc10 dimer indicates that it likely binds two DNA gyres. Harrison presented a model for the yeast centromere in which Ndc10 holds together the two ends of a loop that is only large enough to accommodate a single wrap of DNA around a Cse4 nucleosome. As pointed out by Cleveland, different $\mathrm{CenH} 3$ nucleosomes might exist at different times during the cell cycle and, indeed, CenH3 nucleosomes are found at noncentromeric sites under many circumstances (Henikoff and Furuyama, this volume), including at double-strand breaks, where they lack $\mathrm{H} 2 \mathrm{~A} / \mathrm{H} 2 \mathrm{~B}$ dimers and are required for repair (Zeitlin et al. 2009).

\section{CONCLUSIONS}

Progress made during the past few years in understanding the nucleus has been impressive, with deep new insights that have resolved some perennial issues. Nuclear pores have been mapped with sufficient resolution to un- 
derstand the basics of how they work, and the large-scale organization of nuclear envelope proteins and lamins is evident. The nuclear interior is characterized by diverse self-organizing nuclear bodies and by chromosome territories and chromatin compartments that are contiguous but nevertheless can interact in trans with other sites. Chromatin itself provides enough structural strength when attached to the lamins that there is evidently no need for a fibrillar matrix, and diffusion within a high protein concentration environment seems to be sufficient for regulatory proteins to find their binding sites (Lionnet et al., this volume). There are so many diverse types of heterochromatin and pathways for generating it that the term seems to have lost any meaningful molecular interpretation. Instead, our deepening knowledge of chromatin dynamics provides a better mechanistic basis for understanding epigenetic inheritance that underlies reprogramming in germ cells and stem cells.

The progress that has been made in understanding nuclear organization and function has been driven in large part by genomic and imaging technologies, both of which have been advancing at an unprecedented pace. Fluorescence microscopy can now resolve nucleosomes, and live imaging is becoming routine. Gigabase sequencing at an affordable price is a reality, making it possible to obtain genomic, epigenomic, and transcriptomic information from organisms that otherwise lack infrastructural tools. In his closing sentence of the 38th Symposium Summary, Hewson Swift pointed out that it would take $10^{5}$ years to sequence the human genome, which is an example of why one should be cautious in predicting the future of any endeavor that relies on advancing technology. But, even making a conservative extrapolation, I would venture to guess that by the time of the 100th Symposium, nuclear organization and function will mostly be a solved problem, with its components mapped with molecular detail and its dynamics predictable with high reliability.

Swift's acerbic comments on the state of the chromosome field in 1973 were not entirely negative: "And if it has occurred to you during this conference that our knowledge of the chromosome is fragmentary and grossly incomplete, you might try rereading the CSH Symposium Volume 9, Genes and Chromosomes: Structure and Organization, published in 1941. That volume can serve to remind us that during the past 32 years we have made some progress." If we substitute the word "nucleus" for "chromosome," then a much more upbeat statement can be made in 2010. Our understanding of the nucleus might remain fragmentary and grossly incomplete, but recalling the abysmal state of affairs in understanding much worth remembering regarding nuclear organization and function, I would say that a spectacular amount of progress has been made and the future looks bright.

\section{ACKNOWLEDGMENTS}

I am especially grateful to Jim Haber for contributing the limericks. I also thank other Symposium participants and members of my laboratory for stimulating discussions and the Howard Hughes Medical Institute for support.

\section{REFERENCES}

Brown DD, Sugimoto K. 1974. The structure and evolution of ribosomal and 5S DNAs in Xenopus laevis and Xenopus mulleri. Cold Spring Harb Symp Quant Biol 38: 501-505.

Bungard D, Fuerth BJ, Zeng PY, Faubert B, Maas NL, Viollet B, Carling D, Thompson CB, Jones RG, Berger SL. 2010. Signaling kinase AMPK activates stress-promoted transcription via histone H2B phosphorylation. Science 329: 1201-1205.

Capco DG, Wan KM, Penman S. 1982. The nuclear matrix: Threedimensional architecture and protein composition. Cell 29: 847-858.

Chambon P. 1978. Summary: The molecular biology of the eukaryotic genome is coming of age. Cold Spring Harb Symp Quant Biol 42: 1209-1234.

Conde e Silva N, Black BE, Sivolob A, Filipski J, Cleveland DW, Prunell A. 2007. CENP-A-containing nucleosomes: Easier disassembly versus exclusive centromeric localization. J Mol Biol 370: 555-573.

Davis RL, Weintraub H, Lassar AB. 1987. Expression of a single transfected cDNA converts fibroblasts to myoblasts. Cell 51: 987-1000.

Deal RB, Henikoff JG, Henikoff S. 2010. Genome-wide kinetics of nucleosome turnover determined by metabolic labeling of histones. Science 328: 1161-1164.

Dekker J. 2008. Mapping in vivo chromatin interactions in yeast suggests an extended chromatin fiber with regional variation in compaction. J Biol Chem 283: 34532-34540.

Dekker J, Rippe K, Dekker M, Kleckner N. 2002. Capturing chromosome conformation. Science 295: 1306-1311.

de Wit E, Greil F, van Steensel B. 2007. High-resolution mapping reveals links of HP1 with active and inactive chromatin components. PLoS Genet 3: e38.

Eltsov M, Maclellan KM, Maeshima K, Frangakis AS, Dubochet J. 2008. Analysis of cryo-electron microscopy images does not support the existence of 30 -nm chromatin fibers in mitotic chromosomes in situ. Proc Natl Acad Sci 105: 19732-19737.

Gall JG, Cohen EH, Atherton DD. 1974. The satellite DNAs of Drosophila virilis. Cold Spring Harbor Symp Quant Biol 38: 417-421.

Giles KE, Ghirlando R, Felsenfeld G. 2010. Maintenance of a constitutive heterochromatin domain in vertebrates by a Dicer-dependent mechanism. Nat Cell Biol 12: 94-99.

Gurdon JB, Lane CD, Woodland HR, Marbaix G. 1971. Use of frog eggs and oocytes for the study of messenger RNA and its translation in living cells. Nature 233: 177-182.

Hamiche A, Richard-Foy H. 1998. The switch in the helical handedness of the histone (H3-H4) ${ }_{2}$ tetramer within a nucleoprotein particle requires a reorientation of the $\mathrm{H} 3-\mathrm{H} 3$ interface. $J$ Biol Chem 273: 9261-9269.

Hung T, Chang HY. 2010. Long noncoding RNA in genome regulation: Prospects and mechanisms. RNA Biol 7: 582-585.

Ingouff M, Hamamura Y, Gourgues M, Higashiyama T, Berger F. 2007. Distinct dynamics of HISTONE3 variants between the two fertilization products in plants. Curr Biol 17: 1032-1037.

Kallin EM, Cao R, Jothi R, Xia K, Cui K, Zhao K, Zhang Y. 2009. Genome-wide uH2A localization analysis highlights Bmil-dependent deposition of the mark at repressed genes. PLoS Genet 5: e1000506.

Karpen GH, Schaefer JE, Laird CD. 1988. A Drosophila rRNA gene located in euchromatin is active in transcription and nucleolus formation. Genes Dev 2: 1745-1763.

Kimura H, Tao Y, Roeder RG, Cook PR. 1999. Quantitation of RNA polymerase II and its transcription factors in an HeLa cell: Little soluble holoenzyme but significant amounts of polymerases attached to the nuclear substructure. Mol Cell Biol 19: 5383-5392.

Lieberman-Aiden E, van Berkum NL, Williams L, Imakaev M, Ragoczy T, Telling A, Amit I, Lajoie BR, Sabo PJ, Dorschner $\mathrm{MO}$, et al. 2009. Comprehensive mapping of long-range interactions reveals folding principles of the human genome. Science 326: 289-293.

Margueron R, Justin N, Ohno K, Sharpe ML, Son J, Drury WJ III, Voigt P, Martin SR, Taylor WR, De Marco V, et al. 2009. Role 
of the Polycomb protein EED in the propagation of repressive histone marks. Nature 461: 762-767.

McClintock B. 1951. Chromosome organization and genic expression. Cold Spring Harbor Symp Quant Biol 16: 13-47.

Misteli T. 2001. The concept of self-organization in cellular architecture. J Cell Biol 155: 181-185.

Ooi SL, Henikoff S. 2007. Germline histone dynamics and epigenetics. Curr Opin Cell Biol 19: 257-265.

Pardue ML. 1974. Localization of repeated DNA sequences in Xenopus chromosomes. Cold Spring Harb Symp Quant Biol 38: 475-482.

Ringrose L, Paro R. 2007. Polycomb/Trithorax response elements and epigenetic memory of cell identity. Development 134: 223 232.

Sekulic N, Bassett EA, Rogers DJ, Black BE. 2010. The structure of (CENP-A-H4) reveals physical features that mark centromeres. Nature 467: 347-351.

Singer MF. 1982. SINEs and LINEs: Highly repeated short and long interspersed sequences in mammalian genomes. Cell 28: 433-434.

Vakoc CR, Sachdeva MM, Wang H, Blobel GA. 2006. Profile of histone lysine methylation across transcribed mammalian chromatin. Mol Cell Biol 26: 9185-9195.

Zeitlin SG, Baker NM, Chapados BR, Soutoglou E, Wang JY, Berns MW, Cleveland DW. 2009. Double-strand DNA breaks recruit the centromeric histone CENP-A. Proc Natl Acad Sci 106: $15762-15767$.

Zobeck KL, Buckley MS, Zipfel WR, Lis JT. 2010. Recruitment timing and dynamics of transcription factors at the Hsp70 loci in living cells. Mol Cell 40: 965-975. 


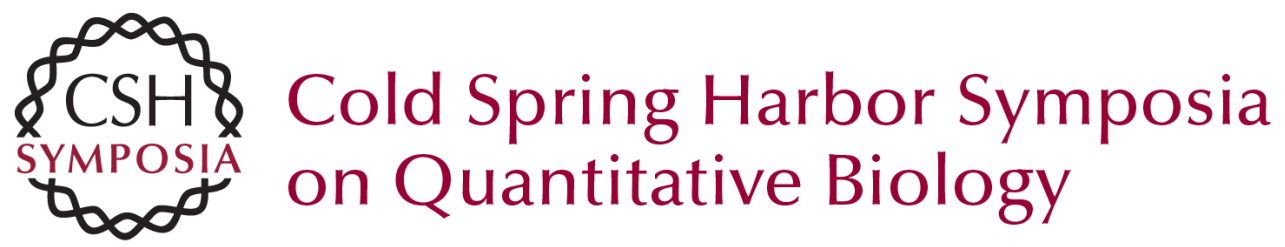

\section{Summary: The Nucleus--A Close-Knit Community of Dynamic Structures}

S. Henikoff

Cold Spring Harb Symp Quant Biol 2010 75: 607-615 originally published online April 18, 2011 Access the most recent version at doi:10.1101/sqb.2010.75.051

References This article cites 32 articles, 18 of which can be accessed free at: http://symposium.cshlp.org/content/75/607.full.html\#ref-list-1

\section{License}

Email Alerting Service top right corner of the article or click here.

To subscribe to Cold Spring Harbor Symposia on Quantitative Biology go to: http://symposium.cshlp.org/subscriptions 